\title{
Effects of Hydrogenized Water on Intracellular Biomarkers for Antioxidants, Glucose Uptake, Insulin Signaling and SIRT 1 and Telomerase Activity
}

\author{
Robert Settineri ${ }^{1, *}$, Jin $\mathrm{Ji}^{2}$, Chunlan $\mathrm{Luo}^{2}$, Rita R. Ellithorpe ${ }^{3}$, Gonzalo Ferreira de Mattos ${ }^{4}$, Steven Rosenblatt ${ }^{5}$, \\ James LaValle ${ }^{6}$, Antonio Jinenez ${ }^{7}$, Shigeo Ohta ${ }^{8}$, Garth L. Nicolson ${ }^{9}$ \\ ${ }^{1}$ Sierra Productions Research, Irvine, USA \\ ${ }^{2}$ Brunswick Laboratories, Inc., Southborough, USA \\ ${ }^{3}$ Tustin Longevity Center, Tustin, USA \\ ${ }^{4}$ Laboratory of Ion Channels, School of Medicine, Universidad de la República, Montevideo, Uruguay \\ ${ }^{5}$ Saint John’s Health Center, Santa Monica, USA \\ ${ }^{6}$ Progressive Medical Center, Orange, USA \\ ${ }^{7}$ Hope4Cancer Institute, Baja California \\ ${ }^{8}$ Department of Biochemistry and Cell Biology, Graduate School of Medicine, Nippon Medical School, Japan \\ ${ }^{9}$ Department of Molecular Pathology, The Institute for Molecular Medicine, Huntington Beach, USA \\ *Corresponding author: sierraprod@aol.com
}

\begin{abstract}
Hydrogen has been shown in several clinical trials to be completely safe without adverse events and there are no warnings in the literature of its toxicity or adverse effects during long-term exposure. Molecular hydrogen has proven useful and convenient as a novel antioxidant and modifier of gene expression in many conditions where oxidative stress and changes in gene expression result in cellular damage. Our intracellular biomarker studies have shown that a hydrogenized water drink formula containing $2.6 \mathrm{ppm}$ dissolved hydrogen was able to penetrate cellular membranes and function as an antioxidant in human liver cells (HePG2) utilizing the Cellular Antioxidant Assay (CAA). This assay uses the protection of a florescent probe as a marker for cellular damage by reactive oxygen species (ROS), such as peroxyl radical, and compares this to the known antioxidant standard, Quercetin. Using this system oxidative damage was reduced in a dose-dependent manner. One $\mathrm{ml}$ of hydrogenized water was found to possess antioxidant capacity equivalent to $0.05 \mu$ mole of quercetin. When examined in a human colon cell line (Caco-2 cells), hydrogenized water demonstrated a dose- and time-dependent permeability inhibition of an intracellular fluorescent glucose derivative (2-NBDG), indicating decreased glucose uptake. In another study, the impact of hydrogenized water on Akt phosphorylation (Ser473), a biomarker for insulin signaling, was monitored in human skeletal muscle cells. The hydrogenized water treatment markedly elevated the level of phosphorylation of Akt (Ser473) in a dose-dependent manner. The anti-aging effects of hydrogenized water were examined utilizing SIRT1 expression as a biomarker of aging in human umbilical cells (HUVECs). Hydrogenized water increased dose-dependent SIRT1 gene expression. Hydrogenized water also increased telomerase activity (an anti-aging biomarker in HUVEC cells) up to 148\% when cells were treated with media containing-25\% hydrogenized water formula. Increased telomerase activity caused by hydrogenized water may be able to protect telomeres from degradation, suggesting the possible use of hydrogenized water in therapeutic interventions of age-related diseases. These studies show that commercial hydrogenized water improved the levels or activities of a few intracellular biomarkers specific for antioxidant activity, glucose uptake, insulin signaling and SIRT 1 and telomerase activities. Industrial Relevance: The molecular hydrogen used in this study indicates that certain commercial sources of hydrogenized water can provide similar antioxidant and gene expression modifications seen in other sources of molecular hydrogen. The biomarkers evaluated here lend well to hydrogenized water's biological activity relating to health conditions and aging.
\end{abstract}

Keywords: Hydrogenized water, bioassays, antioxidant, Caco-2 permeability assay, glucose uptake, insulin receptor, SIRT 1, telomerase activity

Cite This Article: Robert Settineri, Jin Ji, Chunlan Luo, Rita R. Ellithorpe, Gonzalo Ferreira de Mattos, Steven Rosenblatt, James LaValle, Antonio Jinenez, Shigeo Ohta, and Garth L. Nicolson, "Effects of Hydrogenized Water on Intracellular Biomarkers for Antioxidants, Glucose Uptake, Insulin Signaling and SIRT 1 and Telomerase Activity." American Journal of Food and Nutrition, vol. 4, no. 6 (2016): 161-168. doi: 10.12691/ajfn-4-6-4. 


\section{Introduction}

Hydrogen is the smallest and most abundant element, and in its molecular form $\mathrm{H}_{2}$ is colorless, odorless, tasteless and virtually non-toxic. For some time $\mathrm{H}_{2}$ was not considered a bioactive molecule, but a recent study found that inhaled hydrogen gas protects the brain against ischemia reperfusion injury and stroke, and this was likely due to an antioxidant mechanism that reduces free radical damage [1].

Hydrogen can be delivered by gas inhalation, injection of hydrogen-rich saline solutions or by drinking hydrogenrich water (hydrogenized water). Using these methods of administration $\mathrm{H}_{2}$ has been shown to have various preventive and therapeutic effects useful in emergency and critical care medicine [2]. In animal model studies hydrogen gas has been shown to ameliorate intracerebral hemorrhage [3] and reduce hyperoxic lung injury in vivo [4]. Hydrogen gas has also been useful in diagnostic medicine. For example, a hydrogen breath test has been used in clinical practice to detect abnormal bacterial overgrowth in the small intestine, or to diagnose the malabsorption of lactose or fructose [5].

It was reported that molecular hydrogen could improve the damage caused by cerebral ischemia reperfusion injury and selectively reduce strong cytotoxic oxygen radicals, including hydroxyl radical $(\bullet \mathrm{OH})$ and peroxynitrite $\left(\mathrm{ONOO}^{-}\right)$[2,5]. Molecular hydrogen has also proven to be useful-in tissue injury and aging $[1,2,5,7,8,9,10,11]$. In this process, free radicals, such as reactive oxygen species (ROS) and reactive nitrogen species (RNS), are generated as by-products of oxidative metabolism. Since $\mathrm{H}_{2}$ can rapidly diffuse across cell membranes, it is an effective $\cdot \mathrm{OH}$ radical scavenger in cultured cells. ROS/RNS can induce cumulative oxidative damage to cellular macromolecules, eventually resulting in cellular dysfunction, cell death and in some cases, leading to or assisting in the development of various diseases.

It has also been demonstrated that $\mathrm{H}_{2}$ rapidly diffuses across cell membranes and is an effective $\cdot \mathrm{OH}$ radical scavenger in cultured cells and this has been related to its ability to rapidly diffuse across cell membranes. Since then, numerous studies have focused on understanding the potential therapeutic value of hydrogen and hydrogen-rich water in various disease models [12,13].

A comprehensive review the literature on the effects of molecular hydrogen $\left(\mathrm{H}_{2}\right)$ on animals and human subjects and patients with a variety of diagnoses, such as metabolic, rheumatic, cardiovascular, neurodegenerative disease, infections, radiation damage, exercise as well as effects on aging has recently been published. [12].

Here we investigated the effects of hydrogenized water on several intracellular biomarkers related to human health, metabolism and longevity, such as those specific for antioxidant activity weight management/diabetic functional improvement insulin receptor signaling and anti-aging indicators $[17,18]$.

\section{Materials and Methods}

Hydrogenized water identified as "IZUMIO Water" was acquired from Naturally Plus Co. Ltd. Roppongi,
Minato-ku, Tokyo, Japan. At the time of initial packaging, the infused hydrogen within the test water was analyzed by both manometric and diaphragm polarographic electrode measurements at final concentration of $2.6 \mathrm{ppm}$ and $\mathrm{pH}$ 7.0. The human Caco-2 cell line and mouse C2C12 myoblast cell line were obtained from American Type Culture Collection (ATCC, Manassas, USA). HGDMEM (Dulbecco's modified Eagle's medium with high glucose) was from GIBCO ${ }^{\mathrm{TM}}$ (Grand Island, NY, USA). Fetal bovine serum (FBS) and horse serum were purchased from Hyclone (Pittsburg, PA, USA). Insulin, fatty acid-free bovine serum albumin (BSA) was obtained from (Sigma, St. Louis, MO, USA). The sources of other materials are indicated in individual method sections.

\subsection{Cellular Antioxidant Assay}

The Cellular Antioxidant Analysis (CAA) analyzes the capacity of hydrogenized water to protect a fluorescent probe (as a marker) from damage by reactive oxygen species (ROS) in intracellular environments. The assay was carried out as previously described [19]. First, the probe was incubated with representative human liver cells (HePG2 cells obtained from ATCC, Manassas, VA, USA). Once inside cells the loss of probe fluorescence functions as an indication of the extent of damage the probe experiences from the oxygen radicals. Various concentrations of hydrogenized water were incubated with the cells to allow its natural absorption. Then an oxygen radical inducer, 2,2'-Azobis(2-amidinopropane) dihydrochloride (AAPH) from Sigma-Aldrich, (St. Louis, MO, USA), was introduced into cellular environment, which triggers the release of peroxyl radicals. Without excess antioxidants present outside or inside the cells, oxygen radicals permeate through cellular membranes and oxidize the marker probe, and when in excess the oxygen radicals damage the cells. In contrast, when excess antioxidant material is present inside cells, damage does not occur. The cellular antioxidant effect of the hydrogenized water can be measured by assessing the preservation effect of hydrogenized water absorbed inside of cells. quercetin, a known bioeffective antioxidant, is used as the positive control. The cellular antioxidant effect of the test material is compared with that of quercetin, and the comparison result is then expressed as $\mu$ mole quercetin equivalency (QE) per gram (or milliliter) of a tested material.

\subsection{Glucose uptake in Caco-2 human Cells}

This study investigates the effect of hydrogenized water on the permeability of glucose across a Caco- 2 cell monolayer. In this study, Caco-2 human cells were grown at $37^{\circ} \mathrm{C}$ in $5 \% \mathrm{CO}_{2}$ and at $95 \%$ relative humidity using Dulbecco's Modified Essential Medium (DMEM) supplemented with $10 \%$ fetal bovine serum, $1 \%$ non-essential amino acids, and $0.05 \%$ penicillin/streptomycin/amphotericin. Cells were removed at $80-90 \%$ confluence using a $0.25 \%$ trypsin $/ 0.20 \%$ ethylene diamine teraacetic acid (EDTA) solution and replated. Media were changed approximately every $48 \mathrm{~h}$. For the transport experiments Caco-2 cells (passages 26-40) were seeded at $6.5 \times 10^{4}$ cells $/ \mathrm{cm}^{2}$ into polycarbonate 12-well Transwell ${ }^{\circledR}$ plates (Corning Costar Corporation, Cambridge, MA, USA) $(0.4 \mu \mathrm{m}$ mean pore size) and used 21-28 days after seeding. 2-NBDG (2-(N(7-Nitrobenz-2-oxa-1, 3-diazol-4-yl) Amino)-2-Deoxyglucose), 
a fluorescently-labeled deoxy glucose analog, was used as a probe for the detection of glucose movement across Caco-2 cell monolayers. Six concentrations of hydrogenized water $(0,3.125,6.25,12.5,25$ or $50 \%$ v/v hydrogenized water in ionic and osmotic balanced DMEM), along with $200 \mu \mathrm{g} / \mathrm{ml} 2$-NBDG were added to the apical side of confluent monolayers of Caco- 2 cells, and the monolayer cultures were then incubated for $30 \mathrm{~min}$ and $1 \mathrm{hr}$., respectively. At each time point, the receiver (basolateral) sides of the cell media were removed for analysis. The degree of glucose permeation across the cell monolayer was monitored using changes in the fluorescent intensity of 2-NBDG [15]. Statistical assessments were performed by t-test analyses.

\subsection{Cellular Insulin Receptor Signaling Assessment Using Akt phosphorylation}

The effects of hydrogen water on insulin signaling in human skeletal muscle cells was studied using Akt phosphorylation as the cellular biomarker. C2C12 murine myoblasts (ATCC, Manassas, VA, USA, CRL-1772) were cultivated in DMEM (Lonza, Portsmouth, NH, USA) containing $4.5 \mathrm{~g} / \mathrm{L}$ glucose, $2 \mathrm{mM}$ glutamine and $10 \%$ FCS (Thermo Scientific). Cells were plated and grown to approximately 80-90\% confluence. The cells were serum starved (DMEM 0.5\% FCS) overnight, then treated with hydrogenized water at $0,3.125,6.25,12.5,25$ or $50 \% \mathrm{v} / \mathrm{v}$ hydrogenized water in ionic and osmotic balanced DMEM for $60 \mathrm{~min}$. Cells were also treated with concentrations of $30 \mathrm{nM}$ insulin (Sigma, Aldrich, St. Louis, MO, USA) for $15 \mathrm{~min}$ as a control. After the hydrogenized water treatment, cells were then washed in PBS and lysed in lysis buffer (Thermo Fisher Scientific, Fremont, CA, USA). After protein measurement, SDS loading buffer (3\% (w/v) SDS, 3\% (v/v) $\beta$-mercaptoethanol, $10 \mathrm{mM}$ EDTA (pH 8), 20\% (v/v) glycerol, and $0.05 \%(\mathrm{w} / \mathrm{v})$ bromphenol blue) was added, and the samples were boiled for $5 \mathrm{~min}$ at $95{ }^{\circ} \mathrm{C}$. Proteins were separated by SDSpolyacrylamide gel electrophoresis (PAGE) and transferred to a nitrocellulose filter. The membranes were blocked for minimum of $1 \mathrm{~h}$ in Net-G [150 mM NaCl, 5 mM EDTA (pH 8), 50 mM Tris-HCl (pH 7.5), 0.05\% (v/v) Triton X-100, 0.25\% (w/v) gelatin] and incubated with primary antibody overnight at $4{ }^{\circ} \mathrm{C}$. Proteins were detected by horseradish peroxidase-conjugated secondary antibody using an ECL reagent as described by the manufacturer (Thermo Scientific, Fremont, CA, USA).

\subsection{Cellular Aging Assessment}

The impact of hydrogenized water on the cellular aging process was carried out via cellular assessment of two aging biomarkers: SIRT1 and telomerase activity, respectively.

\subsubsection{SIRT1 as Biomarker for Aging}

Cellular aging via SIRT1 assessment measures the ability to prevent or augment cellular SIRT1 production. In this assay, human umbilical vein endothelial cells (HUVECs) were treated with 0, 3.125, 6.25, 12.5, 25 or 50\% $\mathrm{v} / \mathrm{v}$ hydrogenized water in buffer and in duplicate and incubated continued for up to $24 \mathrm{hr}$. One group of cells was treated with $100 \mu \mathrm{M}$ of Resveratrol (Sigma Aldrich,
St. Louis, MO, USA) for $24 \mathrm{hr}$ as control. Subsequently, the cells were lysed, and SIRT1 expression levels were analyzed via Western blot.

\subsubsection{Telomerase Activity as an Aging Biomarker}

Cellular aging via telomerase activity assessment investigates the effect of hydrogenized water on aging via changes in telomerase activities in human umbilical vein endothelial cells (HUVECs). Here, HUVECs were treated with $0,3.125,6.25,12.5,25$ or $50 \% \mathrm{v} / \mathrm{v}$ hydrogenized water in buffer and in duplicate for $24 \mathrm{hr}$. One cell sample was treated with $10 \mathrm{nM}$ raloxifene (Sigma, Aldrich, St. Louis, MO, USA) for $24 \mathrm{hr}$ as positive control. Telomerase activity was determined using the TRAPeze ${ }^{\circledR}$ XL Telomerase Detection Kit (Millipore, Billerica, MA, USA) according to the manufacturer's instructions.

\section{Results and Discussion}

\subsection{Cellular Antioxidant Assay}

CAA analyzes the capacity of a material to protect a fluorescent probe (the biomarker) from damage by reactive oxygen species (ROS) in the intracellular environment. In this assay, peroxyl is used as the free radical (ROS), and human liver cells are used as the cellular model [19]. Quercetin is used as the standard, and the results are expressed as $\mu$ mole quercetin equivalency (QE) per gram (or milliliter) of the tested material. Our analysis determined that the CAA value of the hydrogenized water was $0.05 \mu$ mole QE per $\mathrm{mL}$, which indicates that one $\mathrm{ml}$ of hydrogenized water possesses antioxidant capacity equivalent to $0.05 \mu$ mole of quercetin. This analysis indicates that commercial hydrogenized water can permeate cellular membranes and function as an in vivo antioxidant in human cells. An example of CAA value of a fruit rich in antioxidants, e.g., cranberries, has CAA value of $12.5 \mu$ mole QE per gram of fresh fruit [20]. Compared with cranberries, the CAA value of hydrogenized water is relatively low. However, the usual daily intake of water is much higher than fruit alone. Another point that must be taken into consideration is that the active evaporation of hydrogen from hydrogenized water can significantly impact the actual amount of hydrogen that can permeate into the cells and function as a cellular antioxidant. In human bodies, such hydrogen evaporation may be significantly reduced or the effect of evaporation may be different.

\subsection{Impact of Hydrogenized Water on Glucose Uptake Using the Caco-2 Membrane Model}

Differentiated polarized human Caco-2 colon epithelial cells express a wide range of transporter proteins on their cell membranes that mimic human intestinal epithelia [20]. The Caco-2 absorption study is considered to be the reference standard in pharmaceutical and nutraceutical industries for in vitro prediction of in vivo human intestinal absorption and bioavailability of orally administered substances. In the drug discovery field, the Caco-2 cell permeability assay is also used to predict drug-drug interactions (DDIs) of orally administered drugs 
$[21,22,23]$. Here, we used caco-2 cells as a monolayer epithelial membrane to investigate the bioabsorption of glucose, and how hydrogenized water can impact the degree of glucose absorption.

To study the effect of hydrogenized water on glucose permeation we measured the permeability of 2-NBDG, a fluorescent derivative of glucose, across Caco- 2 cell monolayers after incubation with various concentrations of hydrogenized water (30 or 60 min incubation). The results in Figures 1 and Figure 2 show the ability of hydrogenized water to modulate the permeability of a fluorescent derivative of glucose across Caco-2 cell monolayers in a concentration- and time-dependent fashion, suggesting the potential of molecular hydrogen to function as a glucose permeation inhibitor.

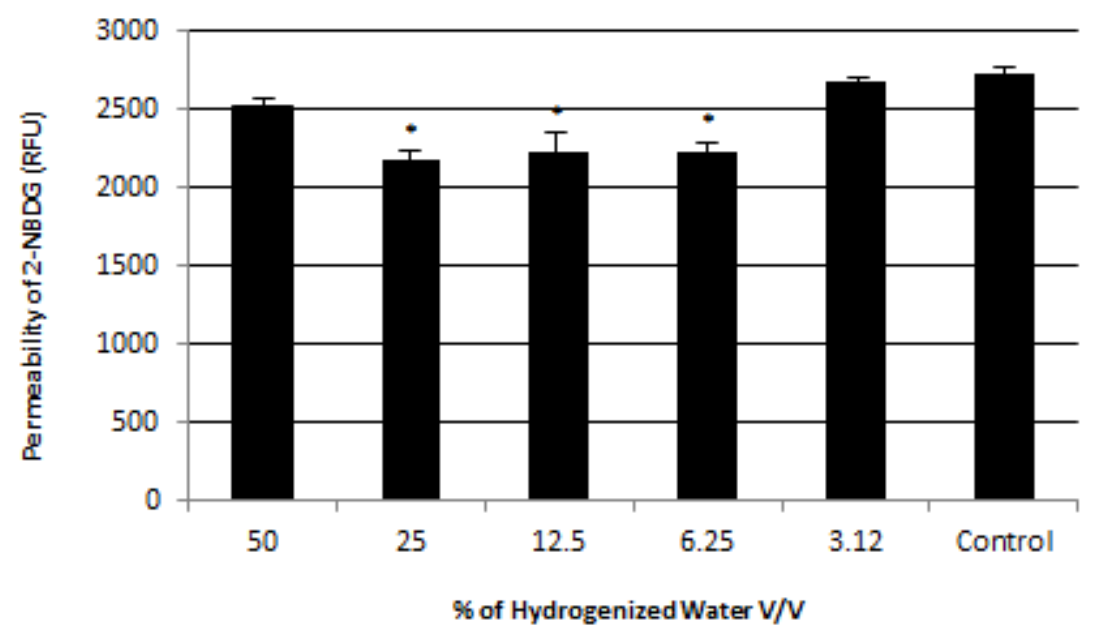

Figure 1. Permeability of glucose (monitored via the fluorescent derivative of glucose 2-NBDG) across Caco-2 cell monolayers upon incubation with HBSS (control) or $0.25,0.5,6.25,12.5,25$ or $50 \% \mathrm{v} / \mathrm{v}$ hydrogenized water in HBSS in an incubation time of $30 \mathrm{~min} . * \mathrm{P}<0.05$

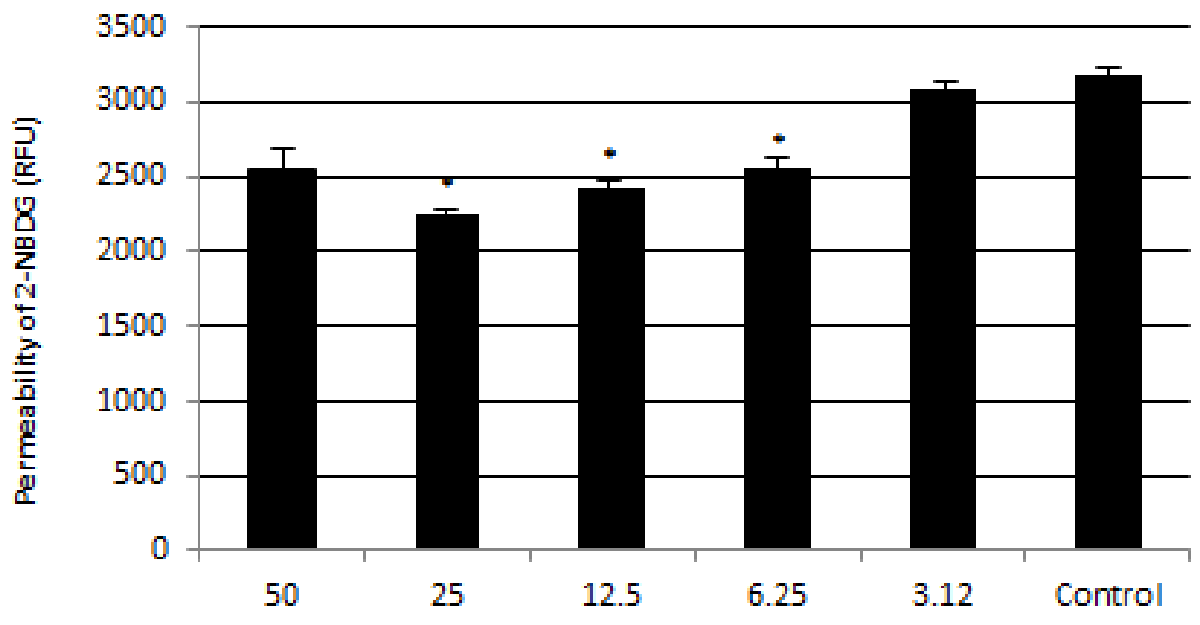

$\%$ of Hydrogenized Water V/V

Figure 2. Permeability of glucose (monitored via a fluorescent derivative of glucose) across Caco-2 cell monolayers upon incubation with HBSS (control) and 3.125\%, 6.25\%, 12.5\%, 25\% and 50\% v/v hydrogenized water in HBSS in an incubation time (60 min). ${ }^{*} \mathrm{P}<0.05$

The results show that the decrease in apical-tobasolateral permeability of glucose in Caco-2 cells (monitored via a fluorescent derivative of glucose) was associated with an increase in hydrogenized water concentration and incubation time (Figure 1 and Figure 2). Incubation of Caco-2 cell monolayers with higher concentrations $(50 \% \mathrm{v} / \mathrm{v})$ of hydrogenized water in HBSS caused a time-dependent decrease in glucose permeability but the effect was less compared to lower concentrations.

\subsection{Impact of Hydrogenized Water on Cellular Insulin Signaling Activity}

Skeletal muscle is the primary site of glucose uptake, disposal, and storage, accounting for approximately 75\% of the entire body's glucose uptake under insulin stimulation. The insulin receptor in this tissue is a transmembrane receptor that is activated by insulin and insulin-like growth factors. Metabolically, the insulin receptor plays a key role in glucose regulation, a functional process that under degenerate conditions may result in various health issues including obesity, metabolic syndrome, diabetes, and cancer. [13]

The main consequence of activation of the insulin receptor is inducing glucose uptake. Under "insulin insensitivity", or a decrease in insulin receptor signaling conditions, cells are unable to take up glucose, resulting in hyperglycemia (an increase in circulating glucose). Eventually this can lead to diabetes mellitus type 2. For this reason, materials that are able to enhance the activation of insulin receptor are helpful in improving insulin sensitivity. When insulin receptor is activated, it 
indirectly activates the phosphorylation of a protein kinase $\mathrm{B}$ (PKB), also known as Akt. Therefore, the measurement of Akt phosphorylation has been used as a surrogate biomarker for insulin receptor activation [24]. In our study, the impact of hydrogenized water on Akt phosphorylation was monitored after cellular treatment with a series of six concentrations of hydrogenized water. Akt is an insulinsignaling transduction molecule existing downstream of the insulin receptor, and its serine residue (Ser473) is phosphorylated by insulin signaling. As shown in Figure 3a and Figure $3 b$, the treatment of hydrogenized water markedly elevated the level of phosphorylation of Akt (Ser473) in skeletal muscle cells in a dose-dependent manner. The level of total Akt has also been measured in a number of cellular studies and shown remaining unchanged throughout the study (Data not shown). A previous study reported that $\mathrm{H}_{2}$ suppressed lipid peroxidation, resulting in a decrease in its end-product, 4hydroxy-2noneal (4HNE), and that 4-HNE modified Akt activation [26]. Thus it is possible the H2 indirectly contributes to insulin signaling [25].

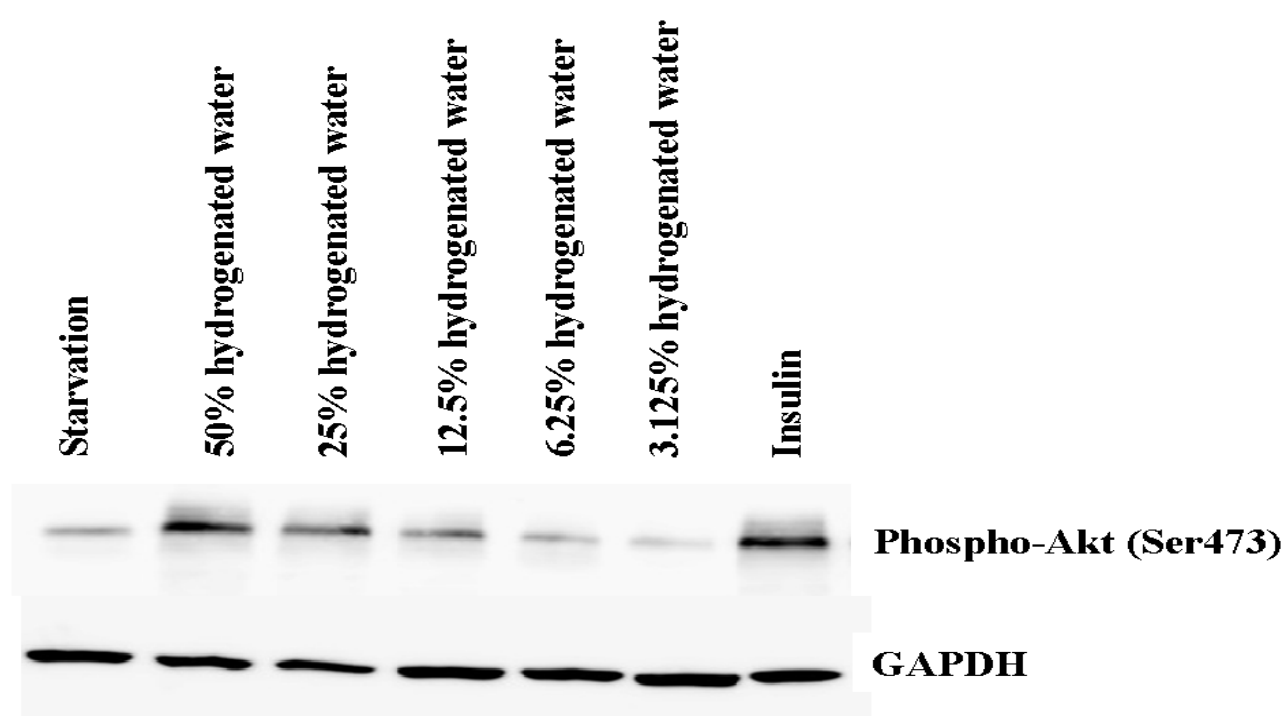

Figure 3(a). Effects of Hydrogenized water on the Akt insulin signaling pathway in skeletal muscle cells. Skeletal muscle cells were incubated with $0 \%$, $3.125 \%, 6.25 \%, 12.5 \%, 25 \%$ and $50 \% \mathrm{v} / \mathrm{v}$ hydrogenized water in buffer for $60 \mathrm{~min}$. Cells were treated with concentrations of $30 \mathrm{nM}$ insulin (Sigma, Aldrich, St. Louis, MO) for $15 \mathrm{~min}$ as control and lysed for phospho-Akt (Ser473) and GAPDH Western blotting. (b) The intensity of the Western blotting images shown in Figure 3(b)

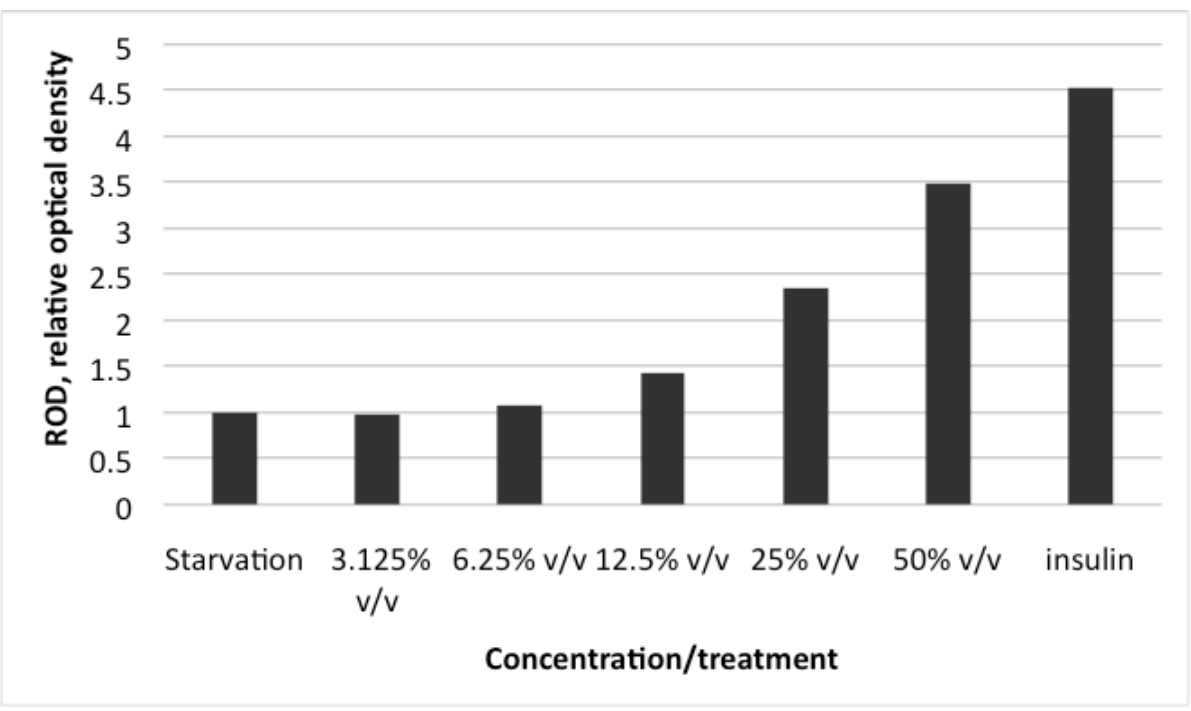

Figure 3(b). The intensity of the optical density of the Western blotting images shown in Figure 3(a)

\subsection{Impact of Hydrogenized Water on Cellular Aging Processes}

Aging is a complex biological process that involves various changes in biochemical activities, among which SIRT1 expression and telomerase activity have been identified as key biomarkers of the aging process. Here, we studied the impact of hydrogenized water on these two key markers to assess the potential anti-aging properties of hydrogenized water.

\subsubsection{SIRT1 as biomarker for aging}

SIRT1 is a protein that is believed to play important roles in longevity and reduction of age-related diseases. Previous studies have shown that when mammals age, SIRT1 expression decreases, whereas induction and activation of SIRT1 has been associated with extended lifespan. These studies have triggered the search for SIRT1 activators that may be used as dietary supplements to promote health and longevity [27]. 
Here, we studied the anti-aging effect of hydrogenized water on SIRT1 expression. We compared SIRT1 expression levels of human cells treated with or without test materials. As shown in Figure 4 and Figure 5, hydrogenized water appeared to increase SIRT1 gene expression in a dose-dependent manner until the effect plateaued at $50 \%$ hydrogenized water-

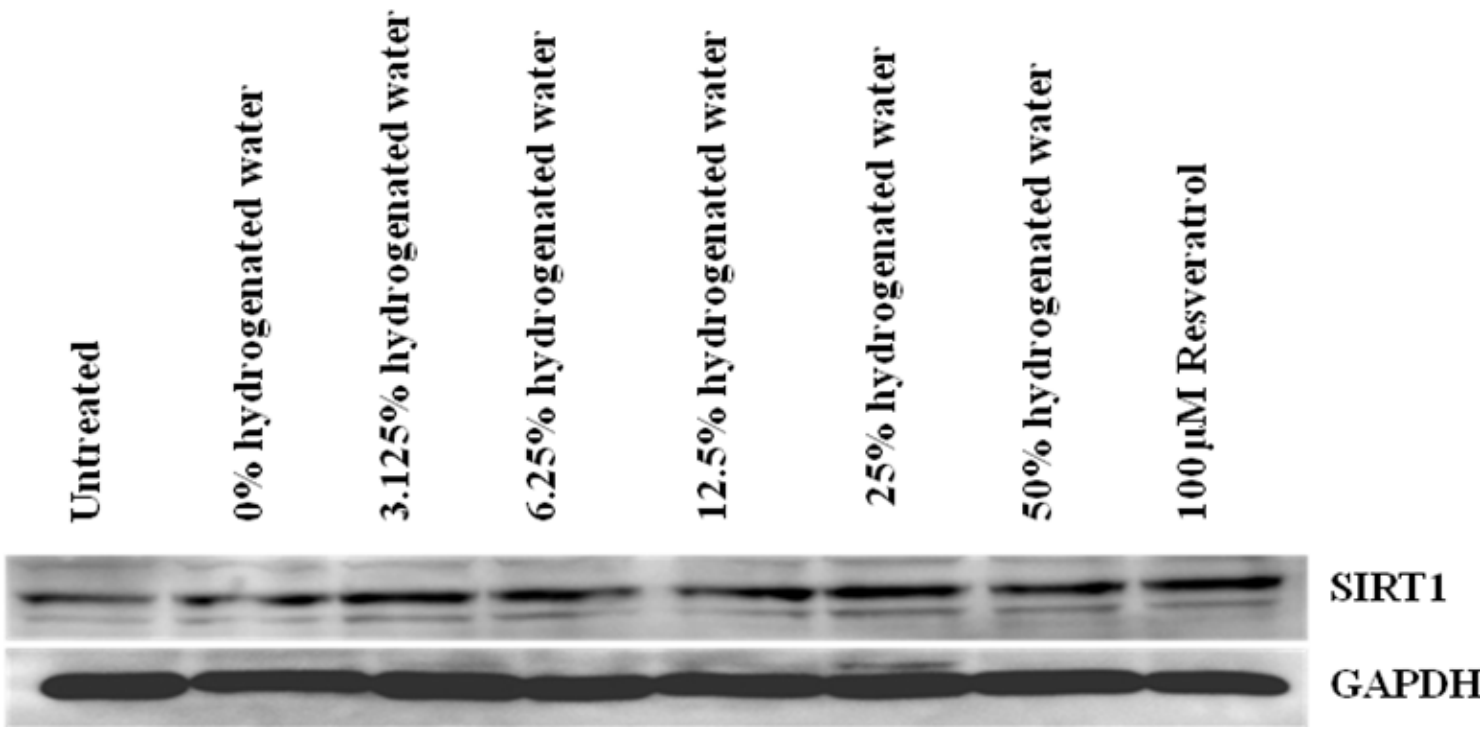

Figure 4. Effects of hydrogenized water on SIRT1 expression in human umbilical vein endothelial cells. Human umbilical vein endothelial cells were incubated with hydrogenized water at 0, 3.125, 6.25, 12.5, 25 and 50\% v/v for 24 hour. Cell were treated with concentrations of $100 \mu \mathrm{M}$ Resveratrol for $24 \mathrm{hr}$ as a control. Cells were lysed and lysates prepared for SIRT1 and GAPDH Western blotting

The Western blotting images shown in Figure 4 were analyzed and the intensity of the images was graphed as Figure 5 below:

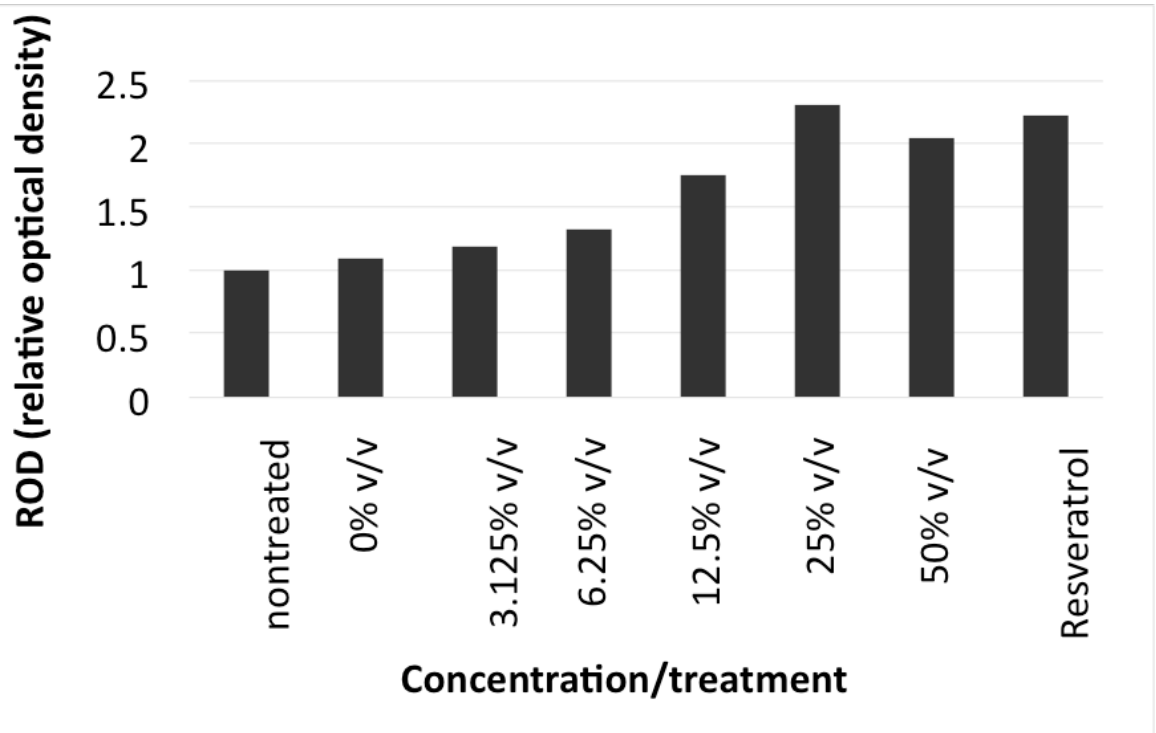

Figure 5. The effect of hydrogenized water on SIRT1 expression in human umbilical endothelial cells. The results are expressed as relative densities of the western blot images in Figure 4

To demonstrate the effect of hydrogenated water on SIRT1 expression, we conducted single run study per concentration for a series of six concentrations of hydrogen water instead of replicates of a single concentration of the hydrogen water. The results indicated that hydrogenized water increased SIRT1 gene expression in a dose-dependent manner in umbilical vein endothelial cells (HUVECs) up to $25 \%$ hydrogenized water. The results also suggested that hydrogenized water may contribute to improvements in regulation of stress response, metabolic homeostasis, and aging. Increased expression of SIRT1 by hydrogenized water treatment could also result in delayed cellular senescence and enhanced endothelial function.

\subsubsection{Telomerase Activity as an Aging Biomarker}

Telomerase is a ribonucleoprotein complex that contributes to the maintenance of telomeric structure and length. The relationship between expression of telomerase, telomere length stabilization or shortening and the extension of the life span of the human cell has recently been reported, making telomerase activity a useful biomarker for anti-aging materials [28]. To assess the impact of hydrogenized water on cellular aging, we studied the effects of hydrogenized water on telomerase 
activity. As shown in Figure 6, hydrogenized water increased telomerase activity in HUVECs when the cells were treated with media containing more than $25 \%$ of hydrogenized water. The results indicate that hydrogenized water may contribute to the improvement of age regulation. Thus increasing telomerase activity by hydrogenized water may be able to protect telomeres from degradation, suggesting a possible utility of hydrogenized water in therapeutic intervention of age-related diseases.

Hydrogenized water increased telomerase activity in representative human cells when cells were treated with media containing $25 \%$ of hydrogenized water. The enhancement of the telomerase biomarker by hydrogenized water was $148.4 \%$ when treated with $25 \%$ hydrogenized water, suggesting that hydrogenized water may be able to protect telomeres from degradation during age-related diseases.

Telomerase activity (normalized to control cells)

\begin{tabular}{|ll|c|}
\hline Reloxifene $\quad 10 \mathrm{nM}$ & 205.0 \\
\hline $\mathrm{H} 2$ water $\quad 50 \% \mathrm{v} / \mathrm{v}$ & 125.0 \\
\hline $\mathrm{H} 2$ water $\quad 25 \% \mathrm{v} / \mathrm{v}$ & 149.4 \\
\hline $\mathrm{H} 2$ water $\quad 12.5 \% \mathrm{v} / \mathrm{v}$ & 101.0 \\
\hline $\mathrm{H} 2$ water $\quad 6.25 \% \mathrm{v} / \mathrm{v}$ & 98.3 \\
\hline $\mathrm{H} 2$ water $\quad 3.125 \% \mathrm{v} / \mathrm{v}$ & 102.4 \\
\hline $\mathrm{H} 2$ water $\quad 0 \% \mathrm{v} / \mathrm{v}$ & 100.0 \\
\hline
\end{tabular}

Figure 6. Effects of Hydrogenized water on Telomerase activity in human umbilical vein endothelial cells (HUVECs). Human umbilical vein endothelial cells (HUVECs) were exposed to $0 \%, 3.125 \%, 6.25 \%$, $12.5 \%, 25 \%, 50 \% \mathrm{v} / \mathrm{v}$ hydrogenized water in bufer, and $10 \mathrm{nM}$ raloxifene for $24 \mathrm{hr} .10 \mathrm{nM}$ raloxifene, a known telomerase activity upregulator, is used as a reference material and positive control. The telomerase activity data are normalized against that of the control cells.

\section{Conclusion}

Molecular hydrogen has proven to be a safe and useful approach to provide antioxidant and gene- and activityregulating activities to cells, tissues and whole organisms. $[12,13]$ Here we found that a commercial hydrogenized water product was effective in providing a selection of these activities to cell systems used for testing the effects of materials as antioxidants and gene- and activityregulating molecules. Although the in vitro systems used here for testing commercial hydrogenized water did not comprise a comprehensive list of all possible test systems, they did show that the commercial hydrogenized water product was active and effective in a few selective tests that have been used previously in product development. Thus we can conclude that the commercial hydrogenized water product tested by us here appears to be bioactive and capable of various activities attributed to molecular hydrogen, such as its antioxidant properties, as well as others that have not been tested previously.

\section{Acknowledgement}

This work is supported by funding provided by Naturally Plus USA.

\section{References}

[1] Ohsawa I, Ishikawa M, Takahashi K, et al. Hydrogen acts as a therapeutic antioxidant by selectively reducing cytotoxic oxygen radicals. Nat Med. 2007; 13: 688-694.

[2] Shen M, Zhang $\mathrm{H}, \mathrm{Yu} \mathrm{C}$, et al. A review of experimental studies of hydrogen as a new therapeutic agent in emergency and critical care medicine. Med Gas Res. 2014; 4:17.

[3] Manaenko A, Lekic T, Ma Q, et al. Hydrogen inhalation ameliorated mast cell-mediated brain injury after intracerebral hemorrhage in mice. Crit Care Med. 2013; 41:1266-1275.

[4] Kawamura T, Wakabayashi N, Shigemura N, et al. Hydrogen gas reduces hyperoxic lung injury via the Nrf2 pathway in vivo. Am J Physiol Lung Cell Mol Physiol. 2013; 304:L646-656.

[5] Ghoshal UC. How to interpret hydrogen breath tests. J Neurogastroenterol Motil. 2011. 17: 312-317.

[6] Ishibashi $\mathrm{T}$, Sato $\mathrm{B}$, Rikitake $\mathrm{M}$, et al. Consumption of water containing a high concentration of molecular hydrogen reduces oxidative stress and disease activity in patients with rheumatoid arthritis: an open-label pilot study. Med Gas Res. 2012; 2(1): 27.

[7] Amitani H, Asakawa A, Cheng K, et al. Hydrogen improves glycemic control in type1 diabetic animal model by promoting glucose uptake into skeletal muscle. PLoS ONE. 2013; 8: e53913.

[8] Gharib B, Hanna S, Abdallahi OM, et al. Anti-inflammatory properties of molecular hydrogen: investigation on parasiteinduced liver inflammation. C. R. Acad. Sci. III. 2001; 324: 719-724.

[9] Lio A, Ito M, Itoh T, et al. Molecular hydrogen attenuates fatty acid uptake and lipid accumulation through downregulating CD36 expression in HepG2 cells. Med Gas Res. 2013; 3(1):6.

[10] Zhang Y, Sun Q, He B, et al. Anti-inflammatory effect of hydrogen-rich saline in a rat model of regional myocardial ischemia and reperfusion. Int J Cardiol. 2011; 148: 91-95.

[11] Song G, Li M, Sang H, et al. Hydrogen-rich water decreases serum LDL-cholesterol levels and improves HDL function in patients with potential metabolic syndrome. J Lipid Res. 2013; 54(7): 1884-1893.

[12] Nicolson G, Ferreira de Mattos G, Settineri R, Costa C, Ellithorpe R, Rosenblatt S, La Valle J, Jimenez A, Ohta S. Clinical Effects of Hydrogen Administration: From Animal and Human Diseases to Exercise Medicine. Int. J. Clin. Med. 2016; 7(1):1-45.

[13] Ohta S. Molecular hydrogen as a preventive and therapeutic medical gas: initiation, development and potential of hydrogen medicine. Pharm Ther. 2014; 144: 1-11.

[14] Wolfe, K. L. and R. H. Liu. Cellular antioxidant activity (CAA) assay for assessing antioxidants, foods, and dietary supplements. $J$ Agricultural and Food Chemistry. 2007; 55(22): 8896-8907.

[15] Zou, C., Y. Wang and Z. Shen. "2-NBDG as a fluorescent indicator for direct glucose uptake measurement. J Biochemical and Biophysical Methods. 2005; 64(3): 207-215.

[16] Shi, Y., H. Yan, P. Frost, J. Gera and A. Lichtenstein. "Mammalian target of rapamycin inhibitors activate the AKT kinase in multiple myeloma cells by up-regulating the insulin-like growth factor receptor/insulin receptor substrate1/phosphatidylinositol 3-kinase cascade. Molecular Cancer Therapeutics. 2005; 4(10): 1533-1540.

[17] Brooks, C. L. and W. Gu. "How does SIRT1 affect metabolism, senescence and cancer. Nature Reviews Cancer. 2009; 9(2): 123-128.

[18] Vaziri, H. and S. Benchimol. "Reconstitution of telomerase activity in normal human cells leads to elongation of telomeres and extended replicative life span. Current Biology. 1998; 8(5): 279-282.

[19] McDowell A, Thompson S, et al. Antioxidant activity of Puha (Sonchus oleraceus L.) as assessed by the cellular antioxidant activity (CAA) assay. Phytotherapy Research 2011; 25(12): 1876-1882.

[20] Brunswick laboratories Inc., Brunswick Laboratories Database for ORAC 6.0 and Cellular antioxidant Assay (CAA), Available: http://www.brunswicklabs.com/tech-library/orac-database-preface [Accessed May 20, 2016].

[21] Siissalo et al. Effect of cell differentiation and passage number on the expression of efflux proteins in wild type and vinblastineinduced Caco-2 cell lines, Eur J Pharm Biopharm. 2007 67(2): 548-54. 
[22] Hubatsch et al. Determination of drug permeability and prediction of drug absorption in Caco-2 monolayers, Nat Protoc. 2007; 2(9): 2111-9.

[23] Calcagno et al. , Comparison of drug transporter levels in normal colon, colon cancer, and Caco-2 cells: impact on drug disposition and discovery. 2006; Mol Pharm. 3(1): 87-9.

[24] Cozzone, D, Froejdoe, S, Disse, E, et al. Isoform-specific defects of insulin stimulation of Akt/proteinkinase B (PKB) in skeletal muscle cells from type 2 diabetic patients. Diebetologia. 2008; 51(3): 512-521.

[25] Kobayashi, M, Matsuda, Y, Iwai, H, et al., Coffee Improves Insulin-Stimulated Akt Phosphorylation in Liver and Skeletal
Muscle in Diabetic KK-A(y) Mice, J Nutritional Science and Vitaminology. 2012; 58(6): 408-414.

[26] Kamimura N, Ichimiya H, Iuchi K, Ohta S. Molecular hydrogen stimulates the gene expression of transcriptional coactivator PGC$1 \alpha$ to enhance fatty acid metabolism. NPJ Aging and Mechanisms of Disease. 2016; 2: 16008.

[27] Allard, J, Perez, E, Zou, S, et al., Dietary activators of Sirt1, Molecular and Cellular Endocrinology. 2009; 299(1): 58-63.

[28] Polychronopoulou, S, Koutroumba, P, Telomere length and telomerase activity: Variations with advancing age and potential role in childhood malignancies, J OF Pediatric Hematology Oncology. 2004; 26(6): 342-350. 